\title{
Frontal lobe hemorrhage in a patient with lenticulostriate artery territory infarction and middle cerebral artery occlusion after recanalization: a case study and literature analysis
}

\author{
Rongfei Wang ${ }^{1,2} \wedge$ \\ ${ }^{1}$ Department of Neurology, Guangdong Provincial Hospital of Chinese Medicine, Guangzhou, China; ${ }^{2}$ The Second School of Clinical Medicine, \\ Guangzhou University of Chinese Medicine, Guangzhou, China
}

Correspondence to: Rongfei Wang. Department of Neurology, Guangdong Provincial Hospital of Chinese Medicine. No. 111 Dade Road, Yuexiu District, Guangzhou, China. Email: wangrongfei30@126.com.

Submitted Aug 17, 2021. Accepted for publication Nov 04, 2021.

doi: 10.21037 /qims-21-817

View this article at: https://dx.doi.org/10.21037/qims-21-817

\section{Introduction}

The risk of reperfusion injury should be considered in the interventional management of cerebrovascular stenosis or occlusion. Although reperfusion can salvage brain tissue from ischemic necrosis, it can also lead to reperfusion injury, such as irreversible severe cerebral edema or hemorrhagic transformation (1).

The lenticulostriate arteries (LSAs), which stem from the middle cerebral artery (MCA), are the main perforators supplying the internal capsule, the basal ganglia, and a part of the corona radiata of the brain. Because LSAs are terminal vessels and lack collateral connections (2), the lenticulostriate territory is a common site of cerebral infarction (3) and hemorrhagic transformation (4). Revascularization of a stenotic or occlusive proximal artery is well known to carry a high risk of bleeding in the lenticulostriate infarct region.

This study describes the case of a patient with an acute infarction of the left corona radiata with MCA horizontal (M1) segment occlusion who experienced hemorrhage in the ipsilateral frontal lobe following intravascular recanalization. This study offers a new perspective on understanding the effect of chronic ischemia on reperfusion injury leading to hemorrhagic transformation.

\section{Case presentation}

A 61-year-old male with a history of myocardial infarction and poorly controlled arterial hypertension suffered a transient attack on the left side of his body on the day before his presentation at the clinic. During the attack, the patient felt dizziness and weakness in his left arm and leg after approximately 10 minutes of walking in a park. He visited the emergency department the next evening, having experienced left facial drop and speech difficulties for 4 hours. A physical examination showed a National Institute of Health Stroke Scale (NIHSS) score of 2. MRI with diffusion-weighted imaging demonstrated acute and multiple focal infarcts in the right corona radiata (Figure 1A). MR angiography revealed an occlusion of the MCA M1 segment, with a residual stump at its origin (Figure 1B).

The patient was given conventional medical treatment, including dual antiplatelet (aspirin $100 \mathrm{mg} /$ day and clopidogrel $75 \mathrm{mg} /$ day, both with a loading dose of $300 \mathrm{mg}$ ) and atorvastatin therapy. Three days later, the patient developed hemiparesis of the left limb with grade 2 muscle strength and a positive Babinski sign; the NIHSS score increased to 8 . Emergency MRI scans of the brain illuminated more infarcts in the same corona radiata region

\footnotetext{
^ ORCID: 0000-0002-6067-8492.
} 

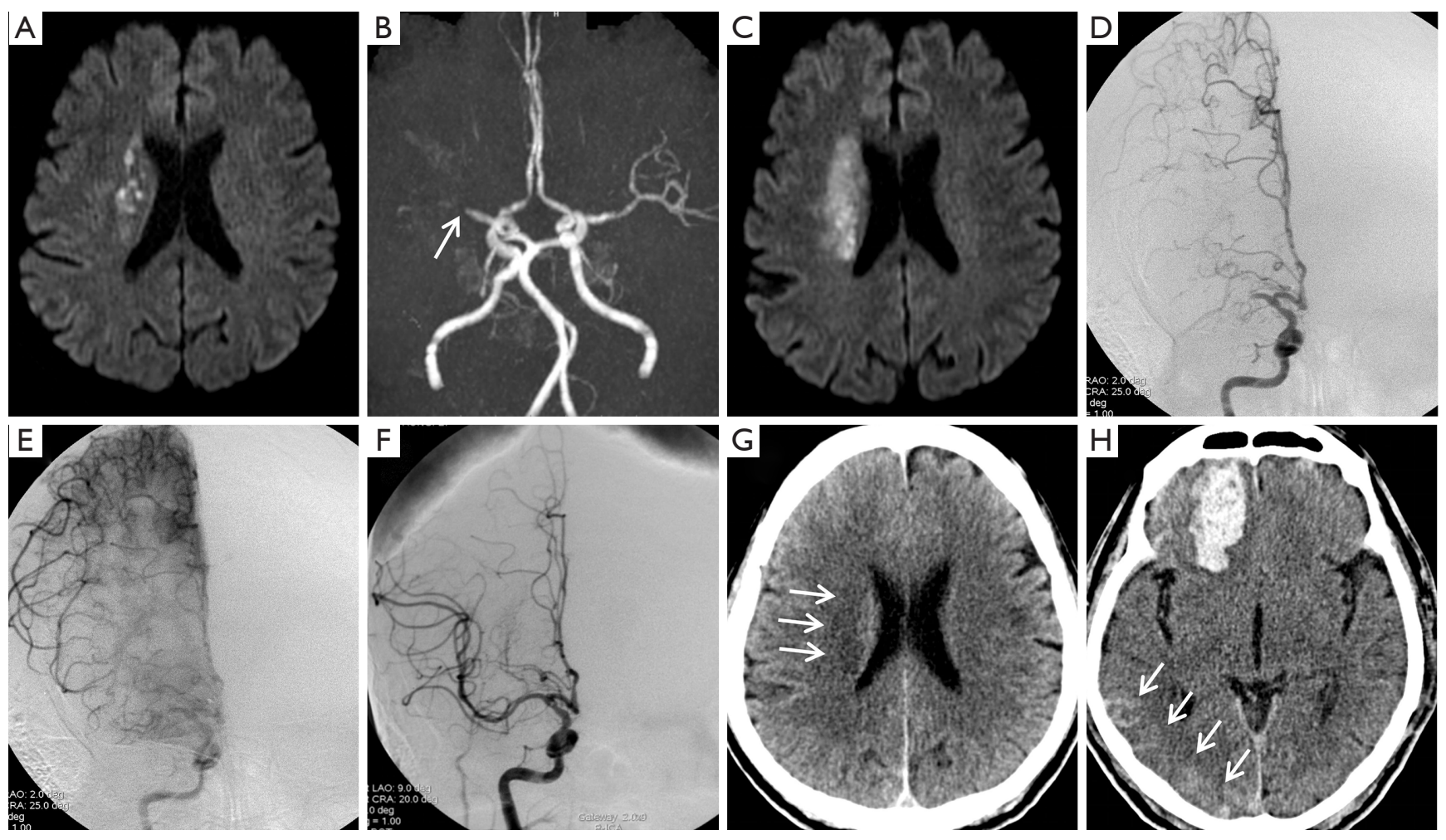

Figure 1 Radiographic findings of bleeding in the non-infarct region after recanalization. (A) Brain MRI scan showing focal infarcts in the right corona radiata. (B) MR angiography image showing occlusion of the M1 segment of the middle cerebral artery (MCA), with a residual stump (white arrow) at its origin. (C) A second MRI scan from 3 days later showing more infarcts in the same corona radiata region. (D) Digital subtraction angiography (DSA) image depicting the occluded MCA at the M1 segment and the robust leptomeningeal arteries connecting the anterior cerebral artery (ACA). (E) A DSA image showing the compensatory collateral flow from the ACA not reversely filling the distal M1 segment of the MCA and the lateral LSAs. The frontal lobe and the frontotemporal watershed region appeared to be in the venous phase, while the basal ganglia and temporal lobe stayed in the arterial phase. (F) Anterograde blood flow in the MCA and imaging of the LSAs were restored immediately after balloon angioplasty (Gateway, 2.0×9.0 mm) to recanalize the blocked M1 segment. (G) CT scan showing no hemorrhage or hyperdensity in the infarcted area (white arrows) 3 hours after treatment intervention. (H) CT scan showing a parenchymal hematoma of approximately $26 \mathrm{~mL}$ in the right frontal lobe and mild hyperdensity in the temporo-occipital cortex (white arrows) $3 \mathrm{~h}$ after the operation.

(Figure 1C). The patient was subsequently diagnosed with progressive ischemic stroke with MCA occlusion.

After signing informed consent, the patient successfully underwent emergent endovascular therapy. Cerebral angiography was performed via a femoral approach. After successful femoral sheath implantation, a bolus of unfractionated heparin was injected intravenously at 3,000 unit and then at 1,000 unit every hour. After general anesthesia administration and heparinization, angiography revealed proximal occlusion of the MCA M1 segment and robust leptomeningeal branch anastomosis of the ipsilateral anterior cerebral artery (ACA) in the right temporal lobe (Figure 1D). Compensatory collateral flow from the ACA did not reversely fill the distal M1 segment and LSAs. The images of the basal ganglia and temporal lobe were slightly delayed compared to those of the ipsilateral frontal lobe and the frontotemporal watershed region (Figure 1E). The occluded M1 segment was then recanalized with the use of a $2.0 \times 9.0 \mathrm{~mm}$ Gateway balloon (Boston Scientific, San Leandro, CA, USA). Under roadmap guidance, an over-thewire balloon was advanced through the lesion with a 0.014 microwire $(200 \mathrm{~cm})$. Balloon angioplasty was performed with the nominal diameter set at 6 atmospheres for 30 seconds. After angioplasty of the stenosis was performed with the Gateway balloon, anterograde blood flow in the MCA and imaging of the LSAs (Figure $1 F$ ) were restored 
immediately.

Three hours after the operation, the patient underwent CT scans after experiencing symptoms of stupor and somnolence. No hemorrhage or hyperdensity was observed in the infarcted area of the corona radiata (Figure 1G). There was, however, a hematoma of approximately $26 \mathrm{~mL}$ in volume in the right frontal lobe parenchyma, with mild hyperdensity observed in the right temporoparieto-occipital junction of the cerebral cortex (Figure 1H). Protamine was not administered for heparin neutralization, because the heparin used during the procedure would have already been naturally metabolized. Due to the low-risk signals on subsequent CT scans, such as the location and volume of the hematoma and no change of blood volume, hematoma evacuation and platelet transfusion therapy were not considered. After 2 weeks of nonsurgical treatment without antiplatelet drugs, the hematoma was absorbed, and the muscle strength of the left limb improved to grade 3.

This research is a retrospective and observational case study. All procedures in this study involving human participants were performed in accordance with the ethical standards of the institutional and/or national research committee(s), and adhered to the Helsinki Declaration (as revised in 2013). Written informed consent was obtained from the patient for the publication of this study and the accompanying images. A copy of the written consent is available for review by the editorial office of this journal.

\section{Discussion}

\section{Vascular structure and autoregulation}

A cortical leptomeningeal network is composed of pial arteries and arterioles at neocortical surface (5). With the help of this network, the ACA and the MCA bring extensive collateral blood flow to their watershed regions. When a pressure gradient occurs in the network due to stenosis or arterial occlusion, these preexisting arterial collaterals are circuited, and then gradually grow over time (6). Even a tiny arteriole can develop into a larger artery and play a significant compensatory role (7). Parenchymal arterioles originate from the arterial network on the surface of the brain parenchyma and plunge perpendicularly into the cortex (5). The vast majority of parenchymal arterioles do not bifurcate on the pial surface before plunging into the parenchyma (8). The walls of arteries and arterioles comprise three typical coaxial coats: the tunica intima, tunica media, and tunica adventitia. The tunica adventitia consists of fibroblasts, collagen fibers, and nerve bundles $(2,9)$. Extracranial, large intracranial, and pial arteries on the brain surface are richly innervated by sympathetic and parasympathetic nerve fibers $(10,11)$ that travel along the tunica adventitia and control vasoconstriction (12).

Cerebral autoregulators include intraluminal pressure, peripheral ions and molecules, and neurotransmitter signals from sympathetic and parasympathetic nerve fibers. Pressure on the vessel walls can immediately affect muscle tone (13), and regulate the structural and functional reorganization of endothelial and smooth muscle cells (14-16). When blood pressure exceeds the limit of myogenic autoregulation, the remaining autoregulation of small arteries and large arterioles depends on the sympathetic autonomic innervation of the tunica adventitia (17). When perivascular sympathetic nerve fibers are denervated, cerebral autoregulation and collateral arteriogenesis become reduced in patients with chronic cerebral hypoperfusion (18). Due to being completely enveloped by astrocytic end-feet (19) and lack of tunica adventitia $(2,20)$, parenchymal arterioles are mostly deprived of sympathetic autoregulation, and less subject to chronic hypoperfusion.

\section{Reperfusion lesions}

Ischemia-reperfusion injury can further damage brain vasculature and parenchyma. Due to the lack of vascular smooth muscle in capillaries, the regulation of capillary flow primarily depends on arteriolar and arterial vascular smooth muscle (21). As autoregulation is impaired by chronic ischemia, these resistant arterioles and arteries expand to their maximum extent under hypoperfusion, and constrict less in the condition of suddenly restored normal intraluminal pressure. Consequently, the capillaries are exposed directly to unbuffered arterial blood pressure (21). As the venous endothelium is part of the blood-brain barrier, the venous component of the blood-brain barrier appears to be the most common site of rupture $(22,23)$ in response to violent conditions, including hypertension (24).

Vasogenic damage leads to "fenestration" in capillary (1). When a certain degree of vasogenic damage occurs, protein components leak from the capillary lumen into the extravascular space, thereby increasing the density of the ischemic region on CT scans (25). Contrast agents may also leak from the lumen into the infarct region when reperfusion occurs. After endovascular treatment, approximately $39 \%$ to $57 \%$ of patients show 
hyperdensity on CT scans, which represents contrast agent leakage $(26,27)$. The non-ionic contrast agents widely used in neuroangiography have molecular weights of approximately 600 to 1,500 Daltons (Da) (28). Both the molecular weight and radius of these agents are between that of macromolecular substances (e.g., albumin, and hemoglobin) and ionic or micromolecules (e.g., $\mathrm{Na}^{+}, \mathrm{K}^{+}$, and $\mathrm{H}_{2} \mathrm{O}$ ). In mild capillary injury, which impacts capillary permeability, contrast agents may leak from the lumen, whereas macromolecular albumin (approximately 6,900 Da) does not. In moderate capillary damage, both contrast and albumin may leak out, but hemoglobin (approximately $65,000 \mathrm{Da}$ ) does not. In severe lesions, the integrity of the endothelial cells in capillary vessels is destroyed, and all components, including hemoglobin, albumin, and contrast agents, can be extravasated, in what is known as hemorrhagic transformation (1).

In this study, postoperative CT scans showed a hematoma in the right frontal lobe and mild hyperdensity in the cortex of the right temporo-occipital watershed area; however, these two areas did not show infarction on MRI scans, nor were there any corresponding clinical symptoms. These findings suggest that vasogenic lesions may result from arterial autoregulation impairment without parenchymal ischemic injury. In the frontotemporal lobe, the robust leptomeningeal arteries played an active role in improving blood supply to the MCA territory prior to endovascular therapy. After recanalization, these collateral arteries with impaired autoregulation dilated significantly, allowing blood to flood the capillaries. Eventually, the integrity of the capillaries was destroyed by excessive blood flow, and hemorrhagic transformation occurred in the right frontal lobe without ischemia. Due to arterial autoregulation impairment, vascular lesions occurred in the temporo-occipital cortex, but to a lesser degree than in the frontotemporal lobe. Consequently, the capillaries transformed into "fenestrated" capillaries, thus allowing the non-ionic contrast agents to leak from the lumen. Contrast agent leakage caused a slight hyperdensity on CT scans.

\section{Impact of reperfusion on LSAs}

The LSAs mostly originate from the M1 segment of the MCA trunk at an almost right angle. The LSAs have an average diameter of $480 \mu \mathrm{m}$ (with a range of 100 to $1,280 \mu \mathrm{m}$ ) and travel an average of 1.50 to $4.35 \mathrm{~mm}$ into the subarachnoid space (the extracerebral LSA segment) before penetrating into the parenchyma (the intracerebral LSA segment) (29). The caliber of the LSAs in the intracerebral segments is mostly $<350 \mu \mathrm{m}$ at the origin, and about 30 $\mu \mathrm{m}$ at the terminal (2). The LSAs are usually divided into medial and lateral arteries, the latter of which typically have bigger calibers and longer extracerebral segments than the former $(2,30)$. Compared with the pial arterial network on the neocortical surface, LSAs have shorter extracerebral segments and lack an arterial network. This implies that sympathetic autoregulation exerts less of an impact on LSAs than on pial arteries and arterioles due to the limited length of the tunica adventitia containing nerve bundles. Therefore, in chronic hypoperfusion caused by proximal arterial stenosis or occlusion, the cumulative dilatation effect on the LSAs is less than that on pial arteries and arterioles. When the stenosis or occlusion is relieved and perfusion resumes, the LSAs are less over-dilated relative to pial arteries and arterioles, resulting in a lesser increase in capillary flow.

In this study, progressive cerebral infarction occurred in the right corona radiata supplied by the lateral LSAs. However, after intravascular recanalization, hemorrhagic transformation occurred in the ipsilateral frontal lobe, which was sufficiently supplied by the ACA. Further, the infarct region did not show contrast agent leakage, which is indicative of reperfusion injury. This phenomenon may be explained as follows. As an extracerebral segment, the pial arteries are richly innervated by sympathetic and parasympathetic nerve fibers $(10,11)$ which influence autoregulation and collateral arteriogenesis in chronic cerebral hypoperfusion (18). Due to the extremely short extracerebral segment of LSAs (29), chronic cerebral hypoperfusion caused by stenosis or occlusion exerts little influence on the autoregulation capability of these arteries, and the cumulative effect of reperfusion on arterial dilatation is limited. In this case, therefore, there was no significant increase in luminal blood flow to the LSAs after angioplasty, and no hemorrhagic transformation occurred in the area of the previous infarct in the corona radiata.

\section{Conclusions}

The effect of chronic cerebral ischemia in the context of reperfusion injury may be more obvious in the neocortical area than in the LSA territory.

\section{Acknowledgments}

Funding: None. 


\section{Footnote}

Conflicts of Interest: The author has completed the ICMJE uniform disclosure form (available at https://dx.doi. org/10.21037/qims-21-817). The author has no conflicts of interest to declare.

Ethical Statement: The author is accountable for all aspects of the work in ensuring that questions related to the accuracy or integrity of any part of the work are appropriately investigated and resolved. All procedures performed in this study involving human participants were in accordance with the ethical standards of the institutional and/or national research committee(s) and with the Helsinki Declaration (as revised in 2013). Written informed consent was obtained from the patient for publication of this case report and accompanying images. A copy of the written consent is available for review by the editorial office of this journal.

Open Access Statement: This is an Open Access article distributed in accordance with the Creative Commons Attribution-NonCommercial-NoDerivs 4.0 International License (CC BY-NC-ND 4.0), which permits the noncommercial replication and distribution of the article with the strict proviso that no changes or edits are made and the original work is properly cited (including links to both the formal publication through the relevant DOI and the license). See: https://creativecommons.org/licenses/by-nc-nd/4.0/.

\section{References}

1. Simard JM, Kent TA, Chen M, Tarasov KV, Gerzanich $\mathrm{V}$. Brain oedema in focal ischaemia: molecular pathophysiology and theoretical implications. Lancet Neurol 2007;6:258-68.

2. Hu R, Feng H. Lenticulostriate Artery and Lenticulostriate-artery Neural Complex: New Concept for Intracerebral Hemorrhage. Curr Pharm Des 2017;23:2206-11.

3. Del Bene A, Makin SD, Doubal FN, Inzitari D, Wardlaw JM. Variation in risk factors for recent small subcortical infarcts with infarct size, shape, and location. Stroke 2013;44:3000-6.

4. Moulin T, Crépin-Leblond T, Chopard JL, Bogousslavsky J. Hemorrhagic infarcts. Eur Neurol 1994;34:64-77.

5. Shih AY, Rühlmann C, Blinder P, Devor A, Drew PJ, Friedman B, Knutsen PM, Lyden PD, Mateo C, Mellander
L, Nishimura N, Schaffer CB, Tsai PS, Kleinfeld D.

Robust and fragile aspects of cortical blood flow in relation to the underlying angioarchitecture. Microcirculation 2015;22:204-18.

6. Liebeskind DS. Collateral circulation. Stroke 2003;34:2279-84.

7. Rongfei Wang, Luankun Weng, Mengzhen Li. Effect of vasa vasorum in cerebrovascular compensation: 2 case reports. Ann Transl Med 2020;8:508.

8. Blinder P, Shih AY, Rafie CA, Kleinfeld D. Topological basis for the robust distribution of blood to rodent neocortex. Proc Natl Acad Sci U S A 2010;107:12670-5.

9. van Mook WN, Rennenberg RJ, Schurink GW, van Oostenbrugge RJ, Mess WH, Hofman PA, de Leeuw PW. Cerebral hyperperfusion syndrome. Lancet Neurol 2005;4:877-88.

10. Hamel E. Perivascular nerves and the regulation of cerebrovascular tone. J Appl Physiol (1985) 2006;100:1059-64.

11. Lincoln J. Innervation of cerebral arteries by nerves containing 5-hydroxytryptamine and noradrenaline. Pharmacol Ther 1995;68:473-501.

12. Kawamura K, Sakata N, Takebayashi S. Neuropeptide Yand vasoactive intestinal polypeptide-containing nerve fibers in the human cerebral arteries: characteristics of distribution. Angiology 1991;42:35-43.

13. Ngai AC, Winn HR. Modulation of cerebral arteriolar diameter by intraluminal flow and pressure. Circ Res 1995;77:832-40.

14. Chien S, Li S, Shyy YJ. Effects of mechanical forces on signal transduction and gene expression in endothelial cells. Hypertension 1998;31:162-9.

15. Chiu JJ, Chien S. Effects of disturbed flow on vascular endothelium: pathophysiological basis and clinical perspectives. Physiol Rev 2011;91:327-87.

16. Kontos HA, Wei EP, Navari RM, Levasseur JE, Rosenblum WI, Patterson JL Jr. Responses of cerebral arteries and arterioles to acute hypotension and hypertension. Am J Physiol 1978;234:H371-83.

17. Beausang-Linder M, Bill A. Cerebral circulation in acute arterial hypertension--protective effects of sympathetic nervous activity. Acta Physiol Scand 1981;111:193-9.

18. Zou C, Hao L, Tian H, Song C, Zhang Y, Zhou H, Liu L. The Effect of Sympathetic Denervation on Cerebral Arteriogenesis After Chronic Cerebral Hypoperfusion. Am J Med Sci 2016;351:616-22.

19. Mulligan SJ, MacVicar BA. Calcium transients in astrocyte endfeet cause cerebrovascular constrictions. Nature 
2004;431:195-9.

20. Cipolla MJ, Li R, Vitullo L. Perivascular innervation of penetrating brain parenchymal arterioles. J Cardiovasc Pharmacol 2004;44:1-8.

21. Kulik T, Kusano Y, Aronhime S, Sandler AL, Winn HR. Regulation of cerebral vasculature in normal and ischemic brain. Neuropharmacology 2008;55:281-8.

22. Mayhan WG, Heistad DD. Permeability of bloodbrain barrier to various sized molecules. Am J Physiol 1985;248:H712-8.

23. Mayhan WG, Heistad DD. Role of veins and cerebral venous pressure in disruption of the blood-brain barrier. Circ Res 1986;59:216-20.

24. Hansson HA, Johansson B, Blomstrand C. Ultrastructural studies on cerebrovascular permeability in acute hypertension. Acta Neuropathol 1975;32:187-98.

25. Weng L, Yao D, Wang R. Unusual images of ischemic stroke with hyperacute spontaneous recanalization: a case report. Ann Transl Med 2020;8:1026.

26. Payabvash S, Qureshi MH, Khan SM, Khan M, Majidi S, Pawar S, Qureshi AI. Differentiating intraparenchymal

Cite this article as: Wang R. Frontal lobe hemorrhage in a patient with lenticulostriate artery territory infarction and middle cerebral artery occlusion after recanalization: a case study and literature analysis. Quant Imaging Med Surg 2022;12(3):2178-2183. doi: 10.21037/qims-21-817 hemorrhage from contrast extravasation on postprocedural noncontrast CT scan in acute ischemic stroke patients undergoing endovascular treatments. Neuroradiology 2014;56:737-44.

27. Cabral FB, Castro-Afonso LH, Nakiri GS, et al. Hyperattenuating brain lesions on CT after ischemic stroke and thrombectomy are associated with final brain infarction. Interv Neuroradiol 2017; 23:594-600.

28. Bourin M, Jolliet P, Ballereau F. An overview of the clinical pharmacokinetics of $\mathrm{x}$-ray contrast media. Clin Pharmacokinet 1997;32:180-93.

29. Djulejić V, Marinković S, Maliković A, Jovanović I, Djordjević D, Cetković M, Todorović V, Milisavljević M. Morphometric analysis, region of supply and microanatomy of the lenticulostriate arteries and their clinical significance. J Clin Neurosci 2012;19:1416-21.

30. Umansky F, Gomes FB, Dujovny M, Diaz FG, Ausman JI, Mirchandani HG, Berman SK. The perforating branches of the middle cerebral artery. A microanatomical study. J Neurosurg 1985;62:261-8. 